\title{
Estudo duplo-cego randomizado da correlação entre radiografia simples e ressonância magnética na avaliação do ângulo crítico do ombro: Reprodutibilidade e curva de aprendizado*
}

\section{Double-Blinded Randomized Study of the Correlation between Simple Radiography and Magnetic Resonance Imaging in the Evaluation of the Critical Shoulder Angle: Reproducibility and Learning Curve}

José Carlos Garcia Júnior ${ }^{10}$ Leandro Sossai Altoe ${ }^{1}$ Rachel Felix Muffareg do Amaral ${ }^{2}$ Andre Yui Aihara ${ }^{2,3}$ Hilton Vargas Lutfi ${ }^{1}$ Marcelo Boulos Dumans Mello ${ }^{1}$

1 Instituto NAEON, São Paulo, SP, Brasil

${ }^{2}$ Diagnósticos da América S/A (Dasa), São Paulo, SP, Brasil

${ }^{3}$ Departamento de Radiologia, Universidade Federal de São Paulo, São Paulo, SP, Brasil
Endereço para correspondência José Carlos Garcia Jr., PhD, Instituto Naeon, Avenida Ibirapuera 2144, cj 82 - São Paulo, SP, 04028-001, Brasil (e-mail: josecarlos@naeon.org.br).

Rev Bras Ortop 2021;56(1):78-82.

\section{Resumo}

Palavras-chave

- manguito rotador

- articulação do ombro

- radiografia

- ressonância nuclear magnética

- reprodutibilidade dos testes
Objetivo Avaliar a confiabilidade da obtenção do ângulo crítico do ombro (ACO) na ressonância magnética (RM) comparada com esse mesmo ângulo obtido por meio de radiografias, e avaliar a curva de aprendizado do método.

Métodos As imagens de radiografias e RMs de 15 pacientes foram avaliadas prospectivamente de forma cega e randômica. O ACO foi medido e comparado entre os grupos e subgrupos.

Resultados A média dos ACOs nas imagens de radiografia foi de $34,61^{\circ} \pm 0,67$, e, na $\mathrm{RM}, 33,85^{\circ} \pm 0,53(p=0,29)$. Não houve diferença estatisticamente significativa. Houve curva de aprendizado progressiva na regressão linear entre os subgrupos, de especializando em ombro a especialista e radiologista.

\footnotetext{
Trabalho desenvolvido no Núcleo Avançado de Estudos em Ortopedia e Neurocirurgia (Naeon) e Diagnósticos da América S/A (Dasa), São Paulo, SP, Brasil.
}

recebido 10 de Março de 2019 aceito

30 de Outubro de 2019

Publicado online

Abril 27, 2020
DOI https://doi.org/ 10.1055/s-0040-1701288. ISSN 0102-3616.
(C) 2020. Sociedade Brasileira de Ortopedia e Traumatologia. All rights reserved.

This is an open access article published by Thieme under the terms of the Creative Commons Attribution-NonDerivative-NonCommercial-License, permitting copying and reproduction so long as the original work is given appropriate credit. Contents may not be used for commercial purposes, or adapted, remixed, transformed or built upon. (https://creativecommons.org/ licenses/by-nc-nd/4.0/)

Thieme Revinter Publicações Ltda., Rua do Matoso 170, Rio de Janeiro, RJ, CEP 20270-135, Brazil 


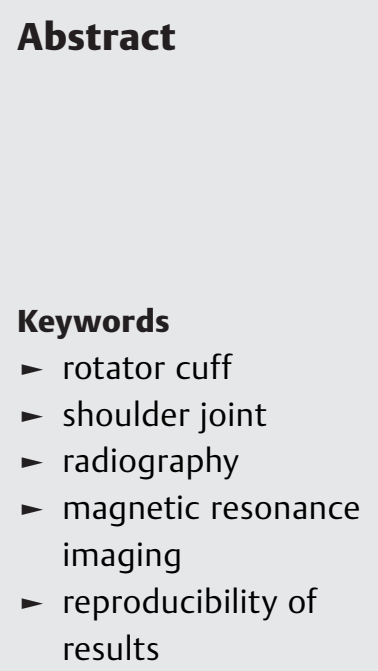

Conclusão Não houve diferença estatisticamente significativa entre o ACO por imagens de radiografia e RM. O método da RM parece ter sua eficiência associada a avaliadores mais experientes. Independente da experiência do avaliador, a variabilidade dos dados foi menor nas avaliações por RM.

Objective To evaluate the feasibility of magnetic resonance imaging (MRI) to obtain the critical shoulder angle (CSA) comparing the results obtained through radiography and MRI, and assess the learning curves.

Methods In total, 15 patients were evaluated in a blinded and randomized way. The CSA was measured and compared among groups and subgroups.

Results The mean angles measured through the radiographic images were of $34.61 \pm 0.67$, and the mean angles obtained through the MRI scans were of $33.85 \pm 0.53(p=0.29)$. No significant differences have been found among the groups. The linear regression presented a progressive learning curve among the subgroups, from fellow in shoulder surgery to shoulder specialist and radiologist.

Conclusion There was no statistically significant difference in the X-rays and MRI assessments. The MRI seems to have its efficacy associated with more experienced evaluators. Data dispersion was smaller for the MRI data regardless of the experience of the evaluator.

\section{Introdução}

A etiologia da tendinopatia do manguito rotador não é ainda completamente conhecida, mas a sobrecarga mecânica é uma das causas mais aventadas para a degeneração tendínea, podendo ter influência de fatores constitucionais dos indivíduos afetados. ${ }^{1-3} \mathrm{O}$ ângulo crítico do ombro (ACO), obtido por meio de avaliações radiográficas, tem sido considerado um importante fator preditivo para essa sobrecarga mecânica. ${ }^{4,5}$ Uma análise em ensaio biomecânico também corroborou o estabelecimento dessa correlação. ${ }^{6}$

O ACO é criticado por alguns autores que não acharam essa mesma correlação; entretanto, um posicionamento inadequado nas radiografias pode ter sido fator limitante nesses estudos. ${ }^{7}$ Baseado na possível fonte de viés de posicionamento do paciente, exames que apresentem imagens de melhor qualidade seriam o caminho lógico para a melhora da reprodutibilidade na avaliação do ACO.

Alguns autores sugeriram uso da tomografia computadorizada, e encontraram alto grau de concordância com o estudo radiográfico. ${ }^{8}$ Entretanto, a tomografía expõe o paciente a doses de radiação mais elevadas do que a radiografia, devendo-se avaliar com mais cautela sua indicação. ${ }^{9} \mathrm{~A}$ ressonância nuclear magnética (RM) não utiliza radiação ionizante, sendo amplamente solicitada para avaliação de diversas condições ortopédicas, e também apresenta menor dependência de fatores posicionais que podem enviesar a imagem radiográfica tradicionalmente utilizada.

Em estudo recente do ACO utilizando a RM, foi sugerida apresentou alta variabilidade de dados quando comparados aos dados obtidos por radiografia, de forma mais evidente nos pacientes com osteoartrose, e que o método não seria adequado. $^{10}$

Este estudo tem como objetivo avaliar a viabilidade do método da RM para a obtenção do ACO, e a correlação entre os resultados obtidos em imagens radiográficas e de RM por nova metodologia de avaliação pela RM.

\section{Materiais e Métodos}

Estudo prospectivo, randomizado, cego e comparativo, para avaliação radiográfica e de RM do ACO, aprovado pelo comitê de ética da instituição com número 2.706 .960 CAAE: 87182318.2.0000.8054.

Os exames de 15 pacientes foram avaliados em ordem randômica e cega para o avaliador. Foram utilizados somente exames de pacientes que iriam ser submetidos tanto à radiografia quanto à RM no mesmo dia e com padronização de posicionamento.

\section{Critérios de Inclusão}

Pacientes com mais de 18 anos de ambos os sexos, que concordaram em participar do estudo, e que apresentavam quaisquer dos sintomas a seguir: perda de força no ombro, instabilidade, limitação do arco de movimento, e dor.

\section{Critérios de Exclusão}

Pacientes com deformidades no ombro, sequelas de fratura no ombro, cirurgias prévias no ombro, erro de posicionamento radiográfico, e indígenas, deficientes mentais ou aqueles de outras populações que apresentem algum conflito ético.

Foi utilizada máquina de RM Espree 1,5 tesla (Siemens, Munique, Alemanha), e o equipamento de radiografia digital MS-18S (General Electric, Boston, MA, EUA). 


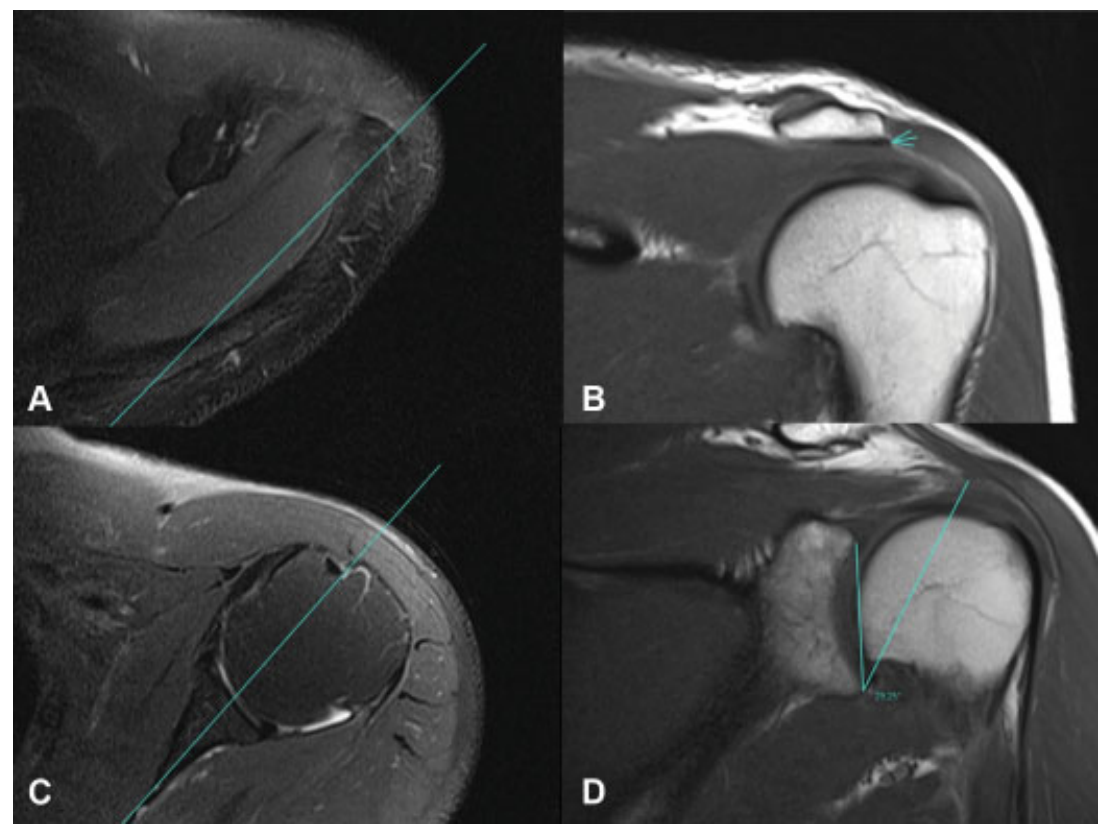

Fig. 1 (A) Marcação do ponto mais lateral do acrômio; imagem em T2, plano axial. (B) Marcação do ponto mais lateral do acrômio; imagem em T1, plano coronal. (C) Marcação do centro da glenoide; imagem em T2, plano axial. (D) Medição do ângulo crítico do ombro (ACO); imagem coronal no corte central da glenoide. Linha entre a borda da glenoide e a projeção para esse corte do ponto mais lateral do acrômio, obtido nos cortes A e B.

O padrão de análise para a posição da radiografia foi anteroposterior verdadeiro, com o paciente na posição ortostática e raios penetrando a $90^{\circ} \mathrm{com}$ a articulação da glenoide. A RM foi realizada com o paciente em decúbito dorsal.

O corte coronal de RM foi estabelecido e padronizado durante o estudo, avaliando-se a melhor visualização das estruturas alvo, e comparando-o com a radiografia.

O ACO foi calculado com ajuda do software Carestream (Carestream Health, Onex Corporation, Toronto, Ontário, Canadá). Após as padronizações, os valores obtidos foram analisados com uso do software STATA (StataCorp., College Station, TX, EUA), versão 15.0.

A medição na RM utilizou imagens em T1 para melhor visualização óssea nos planos axial e coronal (-Figura 1). No plano axial, foi identificado o corte com maior projeção lateral do acrômio, marcado como ponto lateral. No plano axial também foi encontrado o ponto central da cavidade glenoide e marcado no software, a fim de utilizar esse ponto para se estabelecer o corte mais central no plano coronal.

O ponto lateral foi sobreposto a todas as imagens do plano coronal; utilizou-se o corte mais central desse plano para a marcação da reta do eixo superoinferior da cavidade glenoide e da reta entre o ponto mais inferior da glenoide e o ponto lateral, inserido artificialmente na imagem pelo software. $O$ ângulo entre essas duas retas foi considerado o ACO medido na RM.

A medição do ângulo nas radiografias seguiu os padrões descritos por Moor et al ${ }^{4}$ (- Figura 2).

Os dados foram avaliados de forma cega e aleatória por três avaliadores, sendo um especializando em cirurgia do ombro, um especialista em obro com três anos de experiência, e um especialista em radiologia musculoesquelética com três anos de experiência, a fim de estabelecer curva de aprendizado.
A avaliação estatística foi realizada respeitando-se a natureza dos dados. Os resultados foram apresentados no formato de média \pm erro padrão (desvio padrão, DP). Foram considerados significativos valores de $p<0,05$ em uma curva bicaudal. Os exames dos pacientes foram avaliados de forma cega e randômica. Em dados paramétricos, as comparações utilizaram testes $t$ pareados, análise de variância (analysis of variance, ANOVA) e teste de Tukey.

Foi realizada também uma comparação entre as médias obtidas pelos avaliadores e a regressão linear, a fim de se estabelecer diferenças nas curvas de aprendizado da

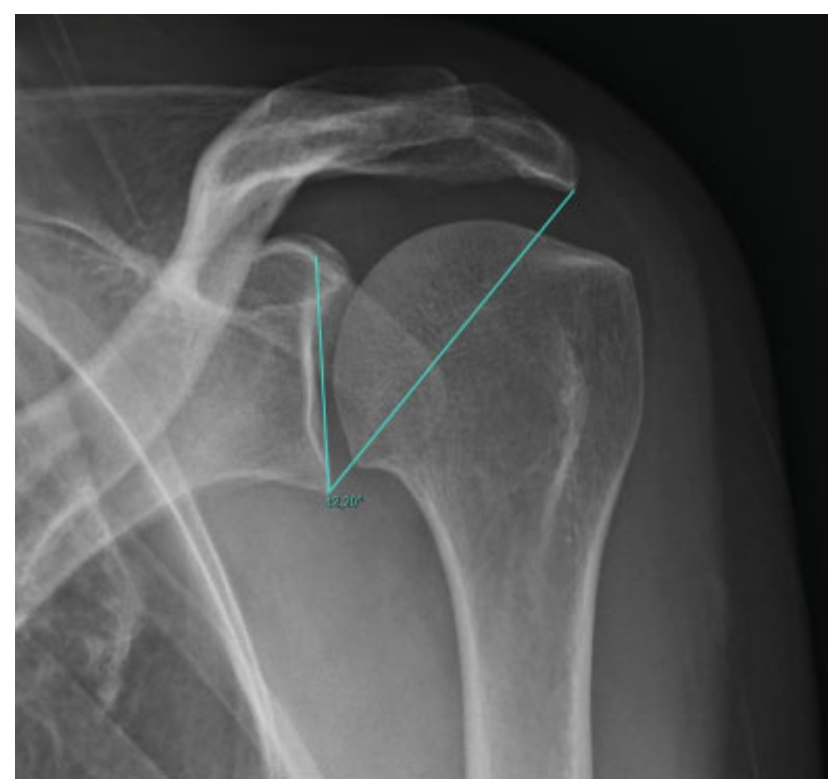

Fig. 2 Medição do ACO na radiografia. 
Tabela 1 Médias com erros padrão dos ângulos por subgrupo

\begin{tabular}{|l|l|l|l|l|}
\hline & Radiografia (RX) & $\begin{array}{l}\text { Ressonância } \\
\text { magnética (RM) }\end{array}$ & $\begin{array}{l}\text { Diferença média } \\
\text { (RX versus RM) }\end{array}$ & $\begin{array}{l}\text { Valor de } p \\
\text { (RX versus RM) }\end{array}$ \\
\hline Especializando em ombro & $35,21^{\circ} \pm 1,32$ & $33,19^{\circ} \pm 0,87$ & $2,02^{\circ}$ & 0,15 \\
\hline Especialista em ombro & $34,43^{\circ} \pm 1,09$ & $33,86^{\circ} \pm 0,92$ & $0,57^{\circ}$ & 0,57 \\
\hline Radiologista & $34,19^{\circ} \pm 1,15$ & $34,49^{\circ} \pm 0,98$ & $0,30^{\circ}$ & 0,84 \\
\hline Análise de variância entre grupos & 0,82 & 0,62 & 0,42 & \\
\hline
\end{tabular}

avaliação de radiografias e da RM entre o especializando e especialista com 3 anos de experiência em cirurgia do ombro.

\section{Resultados}

A média dos ângulos aferidos pelas radiografias foi de $34,61 \pm 0,67$ (DP: 4,54 ), e a média dos exames de RM foi de $33,85 \pm 0,53$ (DP: 3,54); $p=0,29$. A média da diferença entre os ângulos da radiografias e da RM foi de $0,76^{\circ} \pm 0,72$ (DP: 4.81 ).

Os dados e comparações separados nos subgrupos especializando em ombro, especialista em ombro e radiologista estão resumidos na - Tabela $\mathbf{1}$. As comparações entre os grupos pelo método de Tukey estão resumidas na -Tabela 2.

Na regressão linear, a diferença em graus da avaliação entre as radiografias e a RM apresentou constante de $3,07^{\circ}$, com coeficiente de $-1,15^{\circ}$, que é multiplicado por 1 para 0 grupo especializando, por 2 para o grupo especialista, e por 3 para o grupo radiologista.

\section{Discussão}

O ACO tem sido usado na avaliação de pacientes com diversos processos degenerativos e inflamatórios do ombro. Seus dados fornecem uma expectativa que relaciona esse ângulo com alguns tipos de lesões. ${ }^{4}$

Essa avaliação angular, entretanto, não leva em conta as forças de outros músculos como o peitoral maior, o grande dorsal e o bíceps, que também podem contribuir para uma previsibilidade mais precisa das sobrecargas mecânicas do ombro, ${ }^{4-6,11,12}$ visto que simplificações do recrutamento muscular são utilizadas inclusive na sua teorização. ${ }^{11-13}$ Estruturas passivas também não são levadas em conta nessa avaliação; pelos modelos atuais, apenas nos extremos de movimento elas teriam alguma influência nas forças atuantes no ombro. ${ }^{14}$
A avaliação do ACO é feita por meio de exame radiográfico; entretanto, em pacientes já submetidos a RM, o uso dessa radiação ionizante pode ser desnecessário. 0 presente estudo mostra uma tendência adversa à da literatura na comparação das avaliações do ACO por exames radiográficos e RM. ${ }^{10}$ Essa divergência pode ter sua origem nos seguintes erros metodológicos da literatura citada: o ponto mais lateral da clavícula não teve sua marcação adequadamente padronizada, a amostra foi insuficiente, não passando em testes de validação interna, e os exames de RM e radiografia não foram realizados no mesmo momento.

O exame radiográfico pode apresentar maior dificuldade de padronização, sendo mais dependente de variáveis humanas para ser realizado. Esse fato fica claro quando avaliamos a diferenças entre os dados de dispersão em todos os grupos: a dispersão dos dados foi maior nos grupos avaliação radiográfica em relação aos grupos RM independente do tipo de avaliador.

Houve maior concordância e proximidade de dados em examinadores mais experientes, sendo o especialista em radiologia musculoesquelética o que apresentou dados mais próximos, demonstrando que há clara curva de aprendizado, que é mais importante na avaliação por RM. Na ANOVA, há maior concordância na avaliação radiográfica entre os grupos e, tanto pelos resultados demonstrados pela técnica de Tukey quanto pela dispersão de dados e pela regressão linear, há clara curva de aprendizado ligada possivelmente ao maior contato com exames de imagem, principalmente a RM.

A curva de aprendizado da avaliação por RM parece ser mais dependente de treinamento específico do que a curva para avaliação radiográfica. Entretanto, esse fato pode também ter relação com a maior exposição prévia do especializando ao exame radiográfico durante seu treinamento em ortopedia geral, estando ele mais acostumado com avaliações radiográficas do que com imagens de RM.

Tabela 2 Avaliação de Tukey entre grupos e significância das diferenças

\begin{tabular}{|l|l|l|l|}
\hline Tukey & $\begin{array}{l}\text { Valor de } p \text { da } \\
\text { radiografia (RX) } \\
\text { entre grupos }\end{array}$ & $\begin{array}{l}\text { Valor de } p \text { da ressonância } \\
\text { magnética (RM) } \\
\text { entre crupos }\end{array}$ & $\begin{array}{l}\text { Diferença do valor de } \\
p \text { (RX versus RM) } \\
\text { entre grupos }\end{array}$ \\
\hline Radiologiata versus especializando & 0,82 & 0,59 & 0,40 \\
\hline Especializando versus especialista & 0,89 & 0,87 & 0,69 \\
\hline Radiologista versus especialista & 0,99 & 0,88 & 0,87 \\
\hline
\end{tabular}


82 Estudo duplo-cego randomizado da correlação entre radiografia simples e RM Garcia Júnior et al.

Esses efeitos mecânicos não parecem influenciar a extração de imagens.

\section{Conclusão}

Não houve diferença estatisticamente significativa entre os dados da RM e das radiografias para o ACO, com divergência média entre os métodos de apenas $0,76^{\circ}$.

$\mathrm{O}$ método da RM parece ter sua eficiência associada a avaliadores mais experientes.

Independente da experiência do avaliador, a variabilidade dos dados foi menor nas avaliações por RM.

Conflito de interesses

Os autores declaram não haver conflito de interesses.

\section{Referências}

1 Fukuda H, Hamada K, Yamanaka K. Pathology and pathogenesis of bursal-side rotator cuff tears viewed from en bloc histologic sections. Clin Orthop Relat Res 1990;(254):75-80

2 Bedi A, Maak T, Walsh C, et al. Cytokines in rotator cuff degeneration and repair. J Shoulder Elbow Surg 2012;21(02):218-227

3 Hashimoto T, Nobuhara K, Hamada T. Pathologic evidence of degeneration as a primary cause of rotator cuff tear. Clin Orthop Relat Res 2003;(415):111-120

4 Moor BK, Bouaicha S, Rothenfluh DA, Sukthankar A, Gerber C. Is there an association between the individual anatomy of the scapula and the development of rotator cuff tears or osteoarthritis of the glenohumeral joint?: A radiological study of the critical shoulder angle Bone Joint J 2013;95-B(07):935-941

5 Gomide LC, Carmo TC, Bergo GHM, Oliveira GA, Macedo IS. Associação entre o ângulo crítico do ombro e lesão do manguito rotador: um estudo epidemiológico retrospectivo. Rev Bras Ortop 2017;52(04):423-427

6 Gerber C, Snedeker JG, Baumgartner D, Viehöfer AF. Supraspinatus tendon load during abduction is dependent on the size of the critical shoulder angle: A biomechanical analysis. J Orthop Res 2014;32(07):952-957

7 Chalmers PN, Salazar D, Steger-May K, Chamberlain AM, Yamaguchi K, Keener JD. Does the critical shoulder angle correlate with rotator cuff tear progression? Clin Orthop Relat Res 2017;475(06):1608-1617

8 Bouaicha S, Ehrmann C, Slankamenac K, Regan WD, Moor BK. Comparison of the critical shoulder angle in radiographs and computed tomography. Skeletal Radiol 2014;43(08): 1053-1056

9 Smith-Bindman R, Lipson J, Marcus R, et al. Radiation dose associated with common computed tomography examinations and the associated lifetime attributable risk of cancer. Arch Intern Med 2009;169(22):2078-2086

10 Spiegl UJ, Horan MP, Smith SW, Ho CP, Millett PJ. The critical shoulder angle is associated with rotator cuff tears and shoulder osteoarthritis and is better assessed with radiographs over MRI. Knee Surg Sports Traumatol Arthrosc 2016;24(07): 2244-2251

11 Nikooyan AA, Veeger HE, Westerhoff P, Graichen F, Bergmann G, van der Helm FC. Validation of the Delft Shoulder and Elbow Model using in-vivo glenohumeral joint contact forces. J Biomech 2010;43(15):3007-3014

12 Favre P, Snedeker JG, Gerber C. Numerical modelling of the shoulder for clinical applications. Philos Transact A Math Phys. Eng Sci 2009;367(1895):2095-2118

13 Oizumi N, Tadano S, Narita Y, Suenaga N, Iwasaki N, Minami A. Numerical analysis of cooperative abduction muscle forces in a human shoulder joint. J Shoulder Elbow Surg 2006;15(03): 331-338

14 Lippitt S, Matsen F. Mechanisms of glenohumeral joint stability. Clin Orthop Relat Res 1993;(291):20-28 